\title{
Trajectory Similarity Computation based on Interpolation and Integration
}

\author{
Zengwei Zheng \\ School of computing and Computational Science \\ Zhejiang University City College \\ Hangzhou, China \\ zhengzw@zucc.edu.cn
}

Yuanyi Chen

School of computing and Computational Science

Zhejiang University City College
Hangzhou, China
chenyuanyi@zucc.edu.cn

\author{
Wenwang Chen \\ School of Computer Science and Technology \\ Zhejiang University \\ Hangzhou, China \\ wwchen@zju.edu.cn
}

\author{
Dan Chen \\ School of computing and Computational Science \\ Zhejiang University City College \\ Hangzhou, China \\ chend@zucc.edu.cn
}

\begin{abstract}
Trajectory similarity computation is one of the most fundamental functionality, which is applied in many fields, such as trip trajectory mining to find the most popular routes and similar ones, and identify the routes of animal migration and even the stock trends. There are two different kinds of search thoughts, the advanced deep learning method and traditional points matching methods. However, the exiting methods are not totally perfect to solve the trajectory similarity computation problem. The deep- learning method has original problem that it needs a large size of dataset resulting in the requirement of the training time much more than we expected. While the traditional points matching method often suffer from noise and non-uniform sampling rates, because points matching often treats it as two different sequences when the unequal points turn up. In other word, it is often sensitive to the noise which lowers the correct rate of the similarity computation. Based of the statement upon, we propose a new method - applying the interpolation and deformed integration to similarity computation. Experiments shows that our method is robust to the noise and non-uniform sampling rate.
\end{abstract}

Keywords-interpolation; deformed integration; robust; similarity computing; self-similarity;

\section{INTRODUCTION}

With the development of the GPS-enabled devices, trajectory data is being collected over time. In other word, the way we get trajectory data is more and more accessible. Generally, a trajectory is often represented by a sequence of discrete points or locations. And we can map it to a spatial domain, e.g., 2D Euclidean space, which leads to much many of the trajectory similarity computation methods. Computing similarity between two trajectories is fundamental functionality for many applications, such as the migration patterns of animals, identifying hot routes in cities to avoid traffic jam, recommending popular trip routes for travelers and trajectory clustering. As a classic computation problem, a large amount of traditional methods have been proposed, such as longest common sub-sequence (LCSS) [2], edit distance on real sequences (EDR) [4], and dynamic time warping (DTW) [1]. Besides, recently, as the deep learning develops, a method related--t2vec [6], inspired by word2vec, had been proposed.

In most situation, the traditional methods are trying to make points pair match to minimize their distance. But such operation would suffer from some problems, such as the nonuniform sampling rates and noise. For example, due to the devices' inherent limitation, it may fail to grasp the exact location information. What's more, when bypassing the rough area, it is hard for the devices to locate the accurate position. They all cause the generation of the noise. And the devices may lower the frequency of recording out of energy consideration, which leads to the non-uniform and low sampling rates. To cope with the situation, deep-learning-based method had been proposed and achieved remarkable improvement. However, deep learning itself has inherent defect. It needs a large size of dataset. And the training time and the running time is often required much more than our expectation. ${ }^{1}$ To deal with the problems of non-uniform sampling rates, noise and low efficiency, we propose a new method - applying interpolation and deformed integration to trajectory similarity computation. Other than the points matching method, we choose the curve fitting to further research the similarity of different trajectories. The experiment shows us that our method is full of vitality, and it makes noteworthy improvement in both accuracy and efficiency. Overall, the paper makes following contributions:

1) We proposed a trajectory similarity computation model based on the curve fitting. And our method is robust to the nonuniform sampling rates and noise, and computes the similarity in linear time ( we will discuss it in the later part ).

2) We conduct extensive experiments to verify our method outperforming the traditional points matching method in both accuracy and efficiency.

3) We also compare our method with the traditional ones under different proportions of test set and label set. 


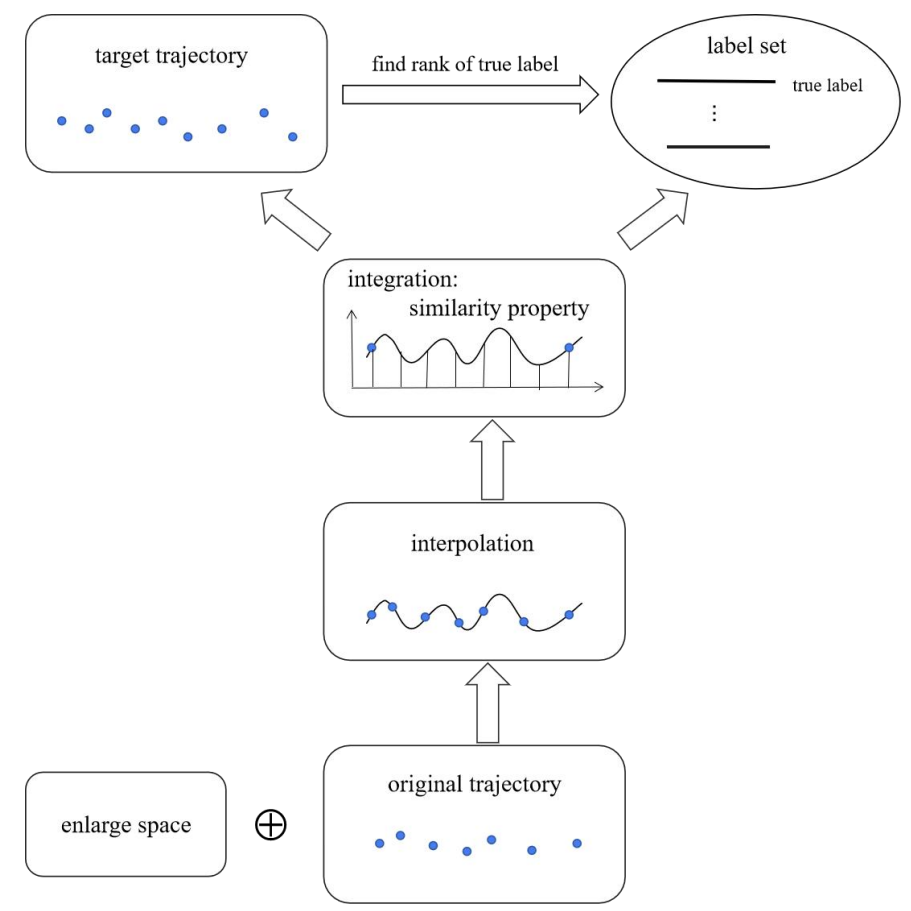

Fig. 1: Framework of proposed method

\section{RELATED WORK}

We briefly review the related work on the trajectory similarity computation. Computing the similarity, or say, the distance between two different trajectories is fundamental functionality in many computing tasks. And it is obvious that the accuracy and the efficiency is what we spot on. DTW [1] was first attempt at tackling the local time shift issue for computing trajectory similarity. T2vec [6] exploits deep representation learning to learn a vector inputted to the encoderdecoder framework to get a trained deep learning model. It highly increased the accuracy of the trajectory similarity computation, but being weak in the efficiency, which is left to be solved. In fact, not only t $2 \mathrm{vec}$ method, almost every deep learning methods such as vRNN[8] share the same inherent defect. They need a large amount of data to train a model, which inevitably results in the long running time. And in some time, the loss of efficiency is unaffordable. APM[7] is another deep learning method. Despite the training time, it is worth mentioning that it solves the issue by learning transition patterns of anchor points from historical trajectories. EDR [4] was proposed to further deal with the problems of the spatial semantics in trajectories. Z. Chen et al. [15] proposed a kind of improved Frechet Distance to deal with the problem of the spatial sequences similarity computation to identify the most popular routes. To improve the robust to the variation in the sampling rates, EDwP[10] was proposed. What's more, EDwP uses the linear interpolation to compute the least loss of the insertion operation, which makes this method identical and inspires our computing measure. And ERP[3] and the modeldriven approach MA[18] were proposed to better capture the spatial features in trajectories. They present different models to speed the proceeding of capturing compared to other methods separately. Wang et al. [13] researched the effect of these similarity computation methods according to their robustness to noise and varying sampling rates.

Moreover, most of the aforementioned existing traditional measures for trajectory similarity computation has time complexity $\mathrm{O}\left(\mathrm{n}^{\wedge} 2\right)$, while our method has the linear time complexity $\mathrm{O}(\mathrm{n}+\mathrm{v})$, where $\mathrm{v}$ represents the average length of trajectories, to measure the similarity, which reflects on the experiment -- our method is much more faster than others.

\section{PROPOSED METHOD}

In this part, we discuss the fundamentals of the proposed method. And then, we will prepare the preliminaries for the experiments. The method's framework is seen in Fig. 1.

\section{A. Interpolation}

Interpolation is a kind of curve fitting method to find a polynomial curve to best fit the discrete points. The definition of the interpolation: in an interval $[a, b]$, given $\mathrm{n}$ discrete points $\left(x_{k}, y_{k}\right), k=1,2, \ldots, n$, for every $\boldsymbol{x}$, we need to find the corresponding $\boldsymbol{y} . f(x)$ is defined in $[a, b]$, and

$$
f\left(x_{k}\right)=y_{k} \quad k=1,2, \ldots, n
$$

And now, what we need to do is to find $g(x)$, for the $\mathrm{n}$ discrete points,

$$
g\left(x_{k}\right)=f\left(x_{k}\right) \quad k=1,2, \ldots, n
$$

We call $g(x)$ an interpolation of $f(x)$ in $[a, b]$. In most cases, polynomial curve is applied when the fitting operation happens. Because for the given discrete points, the polynomial curve is much more convenient to conduct. And the sine-like polynomial curve is more stable because it usually approaches the reality than other fitting methods.

There are some reasons we adopt the interpolation instead of other fitting methods. According to the definition, we can get that the interpolation would absorb all the given discrete points without abandoning any of them. In our experiments, the most of trajectories in the dataset are collections of only dozens of latitude/longitude coordinates. For a complete route, each of them is needed. What's more, one of later operations is to distort some of the points of a trajectory (we will talk about it in detail lately) to simulate the noise in reality. That means it may destroy the simulated environment, or make the excessive operation to desert some points for the fitting.

So in this paper, we adopt Lagrange Interpolation, i.e., a sort of polynomial interpolation. In mathematics, Lagrange interpolation finds a polynomial that happens to take the observed values at each observed point. That means, it can provide a polynomial function that happens to cross all the given points in a two-dimensional plane.

Theorem Polynomials that satisfy the interpolation condition (1) and whose degree does not exceed $n$ exist and unique.

The theorem can be proved in the establishment procedure of the Lagrange polynomial. Let set $D_{n}$ be the collection of angular coordinates of the points $\left(x_{k}, y_{k}\right)$, 
$k=1,2, \ldots, n, D_{n}=\{1,2, \ldots, n\}$, there are $\mathrm{n}$ polynomials $p_{j}(x), \quad j \in D_{n}, \forall k \in D_{n}$,

$$
p_{k}(x)=\prod_{i \in B_{k}} \frac{x-x_{i}}{x_{k}-x_{i}}
$$

Where $B_{k}=\left\{i \mid i \neq k, i \in D_{n}\right\}$. And it is obvious the degree of $p_{k}(x)$ is n-1. Finally, we'll get the Lagrange interpolation:

$$
L_{n}(x)=\sum_{j=1}^{n} y_{j} p_{j}(x)
$$

As the operation above, the Lagrange Interpolation has been conducted and the theorem has been proved. In the later experiments, this interpolation is our important tool to do the fitting operation.

\section{B. Similarity computation}

After the above operation, we have got the fitting polynomial curve on the given several discrete points. Imagine that there are two trajectories, the target $\boldsymbol{T}$ and the compared one, $\boldsymbol{S}$, represented by several latitude/longitude points individually. Using Lagrange Interpolation, we transform the trajectories into two fitting polynomial curves. And now, the problem transforms to how to measure the similarity of this two curves. In this paper, we are trying to apply a deformed integration to compute the similarity.

Conventionally, it is natural to integrate the difference between the two curves. Suppose that this two curves are $T(x)$ and $S(x)$. And the compared trajectory $S$ is recorded from point $\boldsymbol{a}$ to point $\boldsymbol{b}$,

$$
\int_{a \rightarrow b}|T(\mathrm{x})-S(x)| d x
$$

Equation (3) roughly measures the similarity of the two trajectories. Intuitively, the more similar $\boldsymbol{T}$ and $\boldsymbol{S}$ are, the smaller (3) is. It is based on the start and end point of the compared trajectory. Logically, we want to find a trajectory similar to the target. As our experiments set, the compared trajectory is often shorter than the target one.

However, the experiments show that the results of the method above is not very acceptable and fails our original expectation at least. So we go about improving the integration.

There are some abuse in (3). It imports many meaningless features. As we operated before, we applied Lagrange Interpolation to transform the discrete points into the fitting curve. But when we get this step, we actually input countless points irrelevant to the trajectory into the 2-D Euclidean space. And once we adopt (3) as the similarity computation, we just add some meaningless number to the final result, which is proved to influence the result much. Not only in the accuracy, this integration also does not distinct the traditional trajectory similarity computation methods in the efficiency. We need to find the most similar trajectory for the target trajectory in a label set. This integration requires to compute the similarity between the target and the undetermined trajectory dynamically as most the traditional method would do, which leads to time complexity $\mathrm{O}\left(\mathrm{n}^{\wedge} 2\right)$. We need a more efficient method.

Our improvement is as follow. We just consider every single trajectory. And we reserve the Lagrange Interpolation to transform a trajectory $\boldsymbol{T}$ to a fitting curve $T(x)$. To avoid importing other meaningless features, get the span of this trajectory from the start point to the end point and divide it into $m$ parts (1000 as our experiments set), calculate the value corresponding to the interval point on $T(x)$ and sum them all finally. Abstractly, we can regard the sum as the inherent "similarity property" of its corresponding trajectories. And the similarity measure of two trajectories is the absolute value of difference of the respective so-called similarity property, which leads to time complexity $\mathrm{O}(\mathrm{n}+\mathrm{v})$, where $\mathrm{v}$ represents the average length of the trajectories.

Suppose that there are a number of discrete points representing trajectory $\boldsymbol{T}$ in the interval $[p, q]$, and its similarity property is:

$$
\sum_{i=0}^{m-1} T\left(p+\frac{q-p}{m} i\right)
$$

For each trajectory, we can get (4) as the property is inherent. And measuring the similarity depends on the absolute value of the difference of (4). The algorithm is shown in Algorithm 1.

\section{Algorithm 1:}

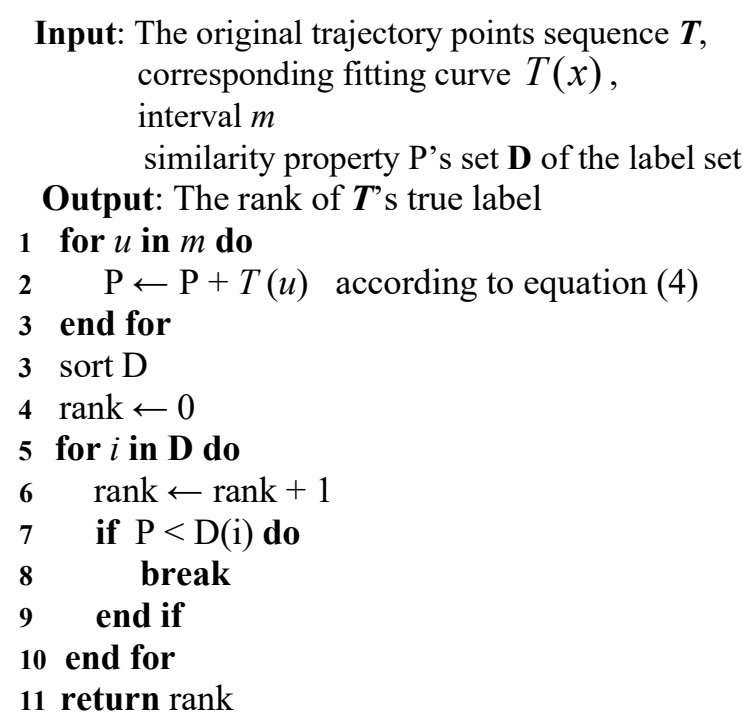

\section{Map trajectory to larger space}

We get the complete measure of the trajectory similarity computation. But for the latitude/longitude space, the prepare is not enough for our experiments. 
In order to pursue the accuracy and efficiency, we lose the stability of the results to some extent. Though such sacrifice is worthy and acceptable, we still want to debauch the loss and enhance the stability as much as possible.

And in this part, we propose an improved method which is to enlarge the space. For the latitude/longitude space, the points representing the trajectories are so intensive as 1 longitude difference is more than $100 \mathrm{~km}$, that when we adopt the Lagrange Interpolation, the fitting curve would flog as the interpolation needs to cross all the discrete points. Flogging makes distortion. To make the fitting curve more smooth, we choose to enlarge the space.

The core rule is to reserve the relative distance between points but to enlarge the absolute distance. For simplicity, select a constant $\alpha$ and $\alpha$ times the distance between the points by fixing the start point of the trajectory. After such operation, we have enlarged the space.

And the experiments show that after enlarging the space, the result is more steady.

\section{Noise and varying sampling rates}

In fact, noise is common in the collected trajectory data. To simulate the actual situation, we need to add the noise to the collected trajectories. Firstly, we confirm a distorting rate $r_{l}$ and randomly select a fraction of the points ( indicated by $r_{l}$ ) and then, distort them by adding a Gaussian noise with a radius 30 (meters). Suppose that a point $(x, y)$ is distorted,

$$
\begin{array}{ll}
x=x+30 \cdot d x, & d x \sim \operatorname{Gaussion}(0,1) \\
y=y+30 \cdot d y, & d y \sim \operatorname{Gaussion}(0,1)
\end{array}
$$

We now have the environment with noise. And another problem -- non-uniform sampling rate is left to cope with.

Due to the reality, such as the devices' inherent flaw and energy concern, we can not always obtain the regular lattice of trajectory points sequence. Likewise, we set the sampling rates.

Before we talk about the sampling rates, suppose that we have a points sequence $\boldsymbol{T},\left\{T^{(i)}\right\}_{i=1}^{n}$, where $\mathrm{n}$ is the length of the points sequence $\boldsymbol{T}$, and we regard it the real route. Then, we create a sample from $\boldsymbol{T}$ by randomly dropping some points with the sampling rates $r_{2}$. For reality concern, we reserve the start point and the end point to avoid changing the underlying route of the sampled trajectory. In our experiments, the sampling rates varies from 0.2 to 0.6 , and we compared with other traditional methods on the basis.

\section{EXPERIMENTS}

We aim to verify the accuracy and efficiency of our proposed method on a taxi dataset. Compared to other three trajectory similarity computation methods which had been proved validly, we want to find our method outperforming in both accuracy and efficiency.

\section{A. Experiment setup}

Dataset: Our experiments are conducted on a real-world taxi dataset (http://www.geolink.pt/ecmlpkdd2015-challenge). It's collected in the city of Porto, Portugal. It contains over 1.7 million trajectories. Each taxi reports its location at 15 second intervals. So each record consists of a series latitude/longitude points representing the trajectory. To reduce the contingency, we remove the trajectories which are less than 30 points. And there are about 1.2 million trajectories left.

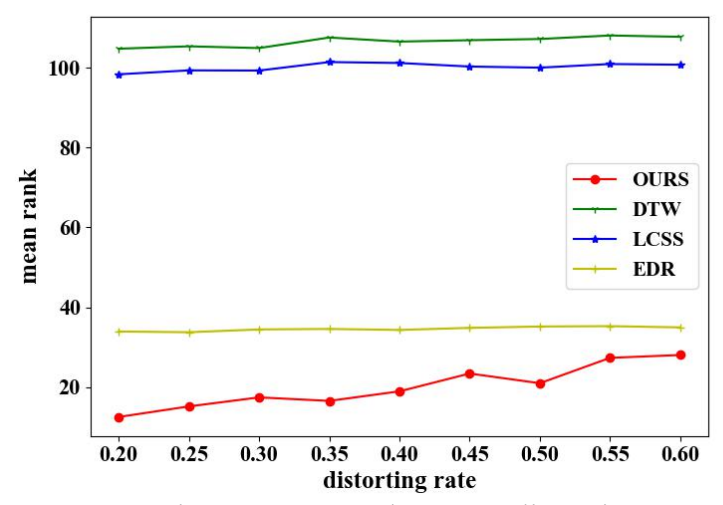

Fig. 2: Mean rank versus distorting rate

Benchmarking Methods: We compare our method with three other traditional methods in accuracy and efficiency, namely DTW[1], LCSS[2], EDR[4]. The above three methods are applied in trajectory similarity computation in most cases. To analyze the performance of our method and other methods in the round, we set many contrast experiments. We fix the sampling rate in 0.6 , and vary the distorting rate in $[0.20,0.25$, $0.30,0.35,0.40,0.45,0.50,0.55,0.60]$. Then, we fix the distorting rate in 0.4 and vary the sampling rate in $[0.20,0.25$, $0.30,0.35,0.40,0.45,0.50,0.55,0.60]$. In every same parameter setting, we try to make our method outstanding.

LCSS and EDR are two of the most widely adopted trajectory measures in spatiotemporal data analyses. LCSS adopts the length of the longest common sub-sequence as the measure of the trajectory similarity computation, while EDR adopts the edit distance. To a certain extent, this two methods are based on the point-to-point match. They seek the as much as more matched points pairs. And obviously, they both abandon the regularity of the points sequence in the $x$ axis, leading to the inaccuracy. And DTW measures the similarity by warping the sequence to best achieve "feature to feature" aligned. But in the trajectory similarity computation, the warping operation may distort the original features of the points sequence, which would also result in the inaccuracy.

Our method proposes a different thinking relative to above three traditional methods. A mixture of the interpolation and "deformed integration" helps to keep the original features and avoid bringing in too many redundant ones.

Evaluation Platform: Our method is implemented in Python running in Windows 10 with an Intel Core i7-7700 CPU.

\section{B. Performance evaluation}

The trajectory dataset we adopt in our experiments is consist of points sequences representing trajectories, which means that the dataset lacks the ground-truth that makes it hard to evaluate the performance of our method and other compared methods in accuracy and efficiency. 
TABLE I: Four methods' mean rank versus sampling rate and distorting rate under 6:4 (a), 7:3 (b) and 8:2 (c) proportions of test set and label set

\begin{tabular}{|c|c|c|c|}
\hline \begin{tabular}{l|l} 
OURS & S. \\
LCSS & $r$ \\
DTW & $a$ \\
EDR & $t$ \\
\cline { 1 - 3 } & $\mathrm{t}$
\end{tabular} & 0.2 & 0.4 & 0.6 \\
\hline 0.2 & $\begin{array}{c}109.30 \\
69.70 \\
148.23 \\
\mathbf{6 2 . 9 8}\end{array}$ & $\begin{array}{l}\mathbf{2 8 . 7 2} \\
64.28 \\
97.41 \\
46.40\end{array}$ & $\begin{array}{l}\mathbf{1 0 . 0 8} \\
61.75 \\
87.27 \\
23.80\end{array}$ \\
\hline 0.4 & $\begin{array}{c}112.01 \\
71.64 \\
149.96 \\
\mathbf{6 3 . 8 8}\end{array}$ & $\begin{array}{c}\mathbf{3 0 . 6 5} \\
66.79 \\
100.35 \\
44.98\end{array}$ & $\begin{array}{l}\mathbf{1 0 . 9 3} \\
62.13 \\
88.46 \\
23.61\end{array}$ \\
\hline 0.6 & $\begin{array}{c}136.77 \\
71.90 \\
151.80 \\
\mathbf{6 2 . 4 8}\end{array}$ & $\begin{array}{c}\mathbf{3 1 . 8 4} \\
66.62 \\
101.56 \\
45.19\end{array}$ & $\begin{array}{l}\mathbf{1 8 . 7 1} \\
59.67 \\
90.10 \\
24.25\end{array}$ \\
\hline
\end{tabular}

(a)

\begin{tabular}{|c|c|c|c|c|}
\hline $\begin{array}{c}\text { OURS } \\
\text { LCSS }\end{array}$ & S. & & & \\
DTW & a & & & \\
EDR & t & & 0.4 & 0.6 \\
\cline { 1 - 4 } D.rate & e & & & \\
\hline & 77.20 & $\mathbf{1 8 . 7 3}$ & $\mathbf{9 . 8 9}$ \\
& & 46.84 & 43.41 & 42.94 \\
0.2 & 122.56 & 76.84 & 66.31 \\
& $\mathbf{4 5 . 1 6}$ & 31.86 & 16.54 \\
\hline & 100.42 & $\mathbf{2 6 . 5 6}$ & $\mathbf{1 0 . 2 8}$ \\
0.4 & 47.16 & 45.60 & 43.69 \\
& 123.36 & 75.10 & 67.57 \\
& $\mathbf{4 5 . 7 8}$ & 31.65 & 16.38 \\
\hline & 98.96 & $\mathbf{3 0 . 2 5}$ & $\mathbf{1 1 . 7 0}$ \\
0.6 & $\mathbf{4 5 . 8 5}$ & 45.24 & 44.06 \\
& 125.23 & 78.59 & 68.69 \\
& 46.67 & 31.92 & 16.40 \\
\hline
\end{tabular}

(b)

\begin{tabular}{|c|c|c|c|c|}
\hline $\begin{array}{c}\text { OURS } \\
\text { LCSS }\end{array}$ & $\begin{array}{c}\text { S } \\
\text { DTW }\end{array}$ & & & \\
DT & 0.2 & 0.4 & 0.6 \\
EDR & t & & & \\
\hline D.rate & $e$ & & & \\
\hline & $\mathbf{3 0 . 1 2}$ & $\mathbf{1 8 . 0 6}$ & $\mathbf{1 1 . 1 2}$ \\
0.2 & 33.09 & 31.63 & 30.18 \\
& 81.48 & 49.69 & 44.12 \\
& 31.68 & 20.65 & 18.15 \\
\hline & 43.92 & $\mathbf{1 9 . 5 8}$ & $\mathbf{1 5 . 4 1}$ \\
0.4 & 34.19 & 31.28 & 31.09 \\
& 85.68 & 51.92 & 44.98 \\
& $\mathbf{3 2 . 5 4}$ & 21.38 & 19.35 \\
\hline & 55.06 & $\mathbf{2 2 . 0 8}$ & $\mathbf{1 5 . 6 8}$ \\
0.6 & 33.94 & 32.37 & 32.01 \\
& 86.70 & 52.66 & 45.79 \\
& $\mathbf{3 2 . 4 3}$ & 24.87 & 20.63 \\
\hline
\end{tabular}

(c)
We adopt a method called self-similarity[6] to perform the evaluation for our experiments. We randomly choose 10,000 trajectories from the dataset and for every sequence in the chosen trajectories, we down-sample and distort them to generate the sub-sequence due to the current sampling rate and distorting rate. And we get the label set of 10,000 trajectories.

And for every trajectory $\boldsymbol{T}$ from another 10,000 trajectories of the dataset, we can also get its sub-sequence $\boldsymbol{S}$ according to the current sampling rate and distorting rate. We call $\boldsymbol{S}$ the label of $\boldsymbol{T}$. Now we get the point pair $(\boldsymbol{T}, \boldsymbol{S})$ and we regard $\boldsymbol{T}$ as the true route in reality, and $\boldsymbol{S}$ as the sampled data collected by the devices. Then we can say that $\boldsymbol{S}$ is the best match for $\boldsymbol{T}$.

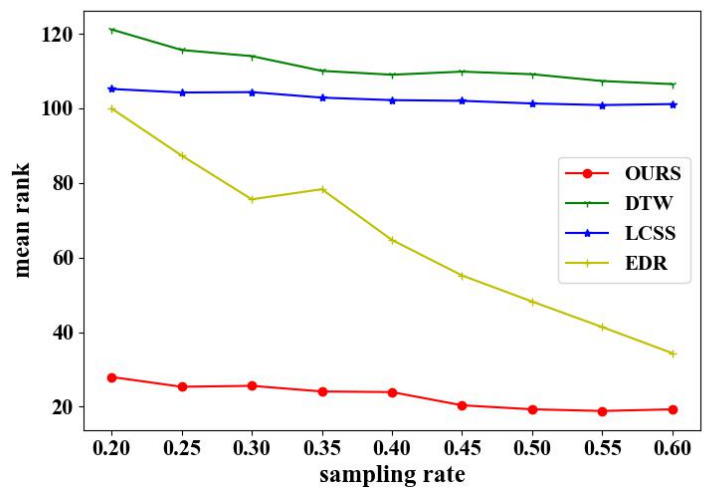

Fig. 3: Mean rank versus sampling rate

A good performance evaluation should keep stable regardless of the sampling or distorting strategy. And the selfsimilarity satisfies the requirement. So the next operation is to hide $\boldsymbol{S}$ in the label set. We try to find the k-nearest neighbors and figure out where the best match $\boldsymbol{S}$ ranks in the whole label set. And we compare the mean-rank of our method to the other three ones.

\section{Experiment in accuracy}

As shown in Fig. 2, when the distorting rate is increasing, every method's mean rank augments generally, which corresponds to reality. The more distort, the more unmatched it gets. Likewise, in Fig. 3, as the sampling rate increases, the mean rank lowers by and large, which means the matching result is getting worse.

The above experiments show that the performance evaluation we adopt -- self-similarity is of stability and it is well adapted in our experiments proceeding. Self-similarity depicts the trend well as the distorting/sampling rate varies and the difference of all the compared methods under the same condition, which all render certain to the self-similarity for experiments.

And our experiments totally satisfy our previous thought. In every distorting rate and sampling rate, our method's mean rank is lower than other three ones, which means our method stands out. To achieve high accuracy, we pay the price of stability to some extent as the Fig shows. And we "enlarge the space" to minimize the cost as we originally wish to. It does work well in our experiments. The curve of our method increases/decreases steadily due to the "enlarge" operation, where we overcome the inherent defect to a large extent. The cost of the stability to enhance the accuracy is worthy.

The above experiments is conducted on basis of 5:5 proportion of the test set and label set. To explore further, we conduct experiments of 6:4, 7:3 and 8:2 proportions, seen in TABLE I, sampling rate denoted as S. rate and distorting rate denoted as D. rate. Due to interpolation, our method performs ordinarily while sampling rate gets low and the fitting curve distorts much, but stands out in most other situations. 


\section{Experiment in efficiency}

Our method aims to stand out in efficiency, too. In the previous method analysis, our method involves "similarity property" in every trajectory, which reduces the time complexity. And we have recorded the running time of the compared methods, seen in Fig. 4.

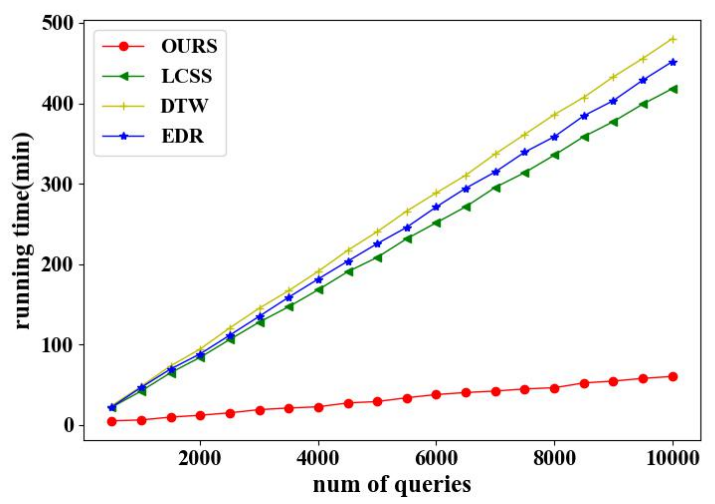

Fig. 4: Comparison of running time

We recorded the running time of our method compared to the traditional methods. And we can get that our method is outperforming in efficiency.

\section{E. Time complexity}

In this part, we discuss the time complexity of our method compared to other traditional trajectory similarity computation methods.

Our method does not dynamically compute the similarity of trajectories. Instead, we introduce a concept of similarity property. And for every single trajectory, we just compute its similarity property. Then, all the trajectories has their own similarity property. So we just need to compare the similarity property to each other and we get the final results. As we analyse before, it is evident that our method's time complexity is $\mathrm{O}(\mathrm{n}+\mathrm{v})$, where $\mathrm{v}$ represents the average length of trajectories.

Our method decreases the time complexity by almost one order of magnitude. But as sacrifice, we lose a little stability to some extent which we view worthwhile.

\section{CONCLUTION AND FUTURE WORK}

In this paper, we propose a trajectory similarity computation method -- a mixture of Lagrange Interpolation and a kind of deformed integration and we compare our method to the traditional similarity computation methods in accuracy and efficiency.

And we set the contrast experiments, which then shows our method stands out in both accuracy and efficiency compared to other methods at the price of stability to some extent. Then, we minimize the sacrifice in stability by the operation of "enlarge" the longitude/latitude space, which also achieves great result.

To evaluate the performance of our method compared to others, we exploit self-similarity, which meets the need of evaluation in performance analysis, such as stability and intuition.
There are some problems remained. On the basis of keeping the satisfactory time complexity, we can explore other measures to compute the similarity property. And we can also change the mean-rank evaluation and the distribution of top- $\mathrm{k}$ trajectories.

\section{FUNDING}

This work is supported by the Young Scientists Fund of the National Natural Science Foundation of China (Grant No. 61802343), Zhejiang Provincial Natural Science Foundation of China (Grant No. LGF19F020019).

\section{REFERENCE}

[1] B.-K. Yi, H. Jagadish, and C. Faloutsos, "Efficient retrieval of similar time sequences under time warping," in $I C D E, 1998$, pp. $201-208$.

[2] M. Vlachos, G. Kollios, and D. Gunopulos, "Discovering similar multidimensional trajectories," in ICDE, 2002, pp. $673-684$.

[3] L. Chen and R. Ng, "On the marriage of lp-norms and edit distance," in PVLDB, 2004, pp. $792-803$.

[4] L. Chen, M. T. Ozsu, and V. Oria, "Robust and fast similarity search for moving object trajectories," in SIGMOD, 2005, pp. $491-502$.

[5] S. Ranu, P. Deepak, A. D. Telang, P. Deshpande, and S. Raghavan,"Indexing and matching trajectories under inconsistent sampling rates," in ICDE, 2015, pp. $999-1010$.

[6] X. Li, K. Zhao, G. Cong, Jensen. C and W. Wei, "Deep Representation Learning for Trajectory Similarity Computation," in ICDE, 2018.

[7] H. Su, K. Zheng, H. Wang, J. Huang, and X. Zhou, "Calibrating trajectory data for similarity-based analysis," in SIGMOD, 2013, pp. $833-844$.

[8] D. Williams and G. Hinton, "Learning representations by backpropagating errors," Nature, vol. 323, no. 6088, pp. 533 - 538, 1986.

[9] H. Jeung, M. L. Yiu, X. Zhou, C. S. Jensen, and H. T. Shen, "Discovery of convoys in trajectory databases," $P V L D B$, vol. 1, no. 1, pp. $1068-$ 1080, 2008.

[10] S. Ranu, P. Deepak, A. D. Telang, P. Deshpande, and S. Raghavan, "Indexing and matching trajectories under inconsistent sampling rates," in ICDE, 2015, pp. 999-1010.

[11] E. Frentzos, K. Gratsias, and Y. Theodoridis, "Index-based most similar trajectory search," in ICDE, 2007, pp. 816-825.

[12] S. Sankararaman, P. K. Agarwal, T. Mølhave, J. Pan, and A. P. Boedihardjo, "Model-driven matching and segm-entation of trajectories," in SIGSPATIAL, 2013, pp. 234-243.

[13] H. Wang, H. Su Datab, K. Zheng, S. Sadiq, and X. Zhou, "An effectiveness study on trajectory similarity mea-sures," in Aliaustranase Conference, 2013, pp. 13-22.

[14] D. Bahdanau, K. Cho, and Y. Bengio, "Neural machine translation by jointly learning to align and translate," arXiv preprint arXiv:1409.0473, 2014.

[15] Z. Chen, H. T. Shen, and X. Zhou, "Discovering popular routes from trajectories," in $I C D E, 2011$, pp. 900-911.

[16] T. Mikolov, I. Sutskever, K. Chen, G. S. Corrado, and J. Dean, "Distributed representations of words and phrases and their compositionality," in NIPS, 2013, pp. 3111-3119.

[17] J. Chung, C. Gulcehre, K. Cho, and Y. Bengio, "Empirical evaluation of gated recurrent neural networks on sequence modeling," arXiv preprint arXiv:1412.3555, 2014.

[18] S. Sankararaman, P. K. Agarwal, T. Mølhave, J. Pan, and A. P. Boedihardjo, "Model-driven matching and segmentation of trajectories," in SIGSPATIAL, 2013, pp. 234-243.

[19] K. Cho, B. Van Merrienboer, C. Gulcehre, D. Bahdanau, F. Bougares, "H. Schwenk, and Y. Bengio, "Learning phrase representations using rnn encoder-decoder for statistical machine translation," arXiv preprint arXiv:1406.1078, 2014

[20] S. Jean, K. Cho, R. Memisevic, and Y. Bengio, "On using very large target vocabulary for neural machine translation," arXiv preprint arXiv:1412.2007, 2014. 